\title{
Introduciendo el enfoque ‘¿Cómo llega a ser representado el problema?"
}

\section{Introducing the 'What's the Problem Represented to be?' approach}

\section{Carol Bacchi²}

Universidad de Adelaida, Australia

El enfoque ¿Cómo llega a ser representado el problema? (CRP) ${ }^{3}$ es un recurso o herramienta destinado a facilitar la interrogación crítica de las políticas públicas. Parte de la premisa de que lo que uno se propone hacer sobre algo, revela lo que uno piensa que es problemático (y que necesita cambiar). Siguiendo este pensamiento, las políticas y las propuestas de políticas contienen representaciones implícitas de lo que se considera el "problema" (representaciones del problema). Por ejemplo, si se recomiendan formas de capacitación para mejorar la condición de la mujer y sus oportunidades de promoción, podemos deducir que su falta de capacitación es el 'problema'.

\footnotetext{
' Artículo original titulado "Introducing the 'What's the Problem Represented to be?' approach", publicado por Carol Bacchi en el libro "Engaging with Carol Bacchi: Strategic Interventions and Exchanges", editado por Angelique Bletsas y Chris Beasley y publicado por la Universidad de Adelaida en 20 I2. El libro puede descargarse gratuitamente en este enlace: https://www.adelaide.edu.au/press/titles/engaging. Agradecemos a la autora y a Toni Luppino, oficial de la biblioteca de la Universidad de Adelaida, por conceder permiso de traducción y republicación del manuscrito.

${ }^{3}$ El nombre del enfoque en inglés es "What's the Problem Represented to be?" y se abrevia WPR.
} 
La tarea en el análisis CRP es leer las políticas con miras a discernir cómo se representa el 'problema' dentro de ellas y someter esta representación a un análisis crítico. Esta tarea se logra a través de un conjunto de seis preguntas y un compromiso adjunto para aplicar las preguntas a las propias propuestas:

1. ¿Cuál es el 'problema' (por ejemplo ‘jugadores problemáticos', 'uso / abuso de drogas', 'desigualdad de género', 'violencia doméstica', 'calentamiento global', 'acoso sexual', etc.) representado en una política o propuesta de política específica?

2. ¿Qué presuposiciones o supuestos sustentan esta representación del 'problema’?

3. ¿Cómo ha surgido esta representación del 'problema'?

4. ¿Qué queda no problematizado en esta representación del 'problema’? ¿Dónde están los silencios? ¿Se puede pensar el 'problema' de otra manera?

5. ¿Qué efectos produce esta representación del 'problema'?

6. ¿Cómo / dónde se ha producido, difundido y defendido esta representación del 'problema'? ¿Cómo ha sido (o podría ser) cuestionada, alterada y reemplazada esta representación del problema?

\section{Aplique esta lista de preguntas a sus propias representaciones de problemas.}

La pregunta 1 ayuda a aclarar la representación implícita del problema dentro de una política o propuesta de política específica. Las preguntas posteriores fomentan:

- una reflexión sobre las premisas subyacentes en esta representación del 'problema' (pregunta 2).

- una consideración de las prácticas y procesos contingentes a través de los cuales ha surgido esta comprensión del 'problema' (pregunta 3).

- un escrutinio cuidadoso de posibles lagunas o limitaciones en esta representación del 'problema', acompañado de una imaginación inventiva de alternativas potenciales (pregunta 4). 
- una evaluación de cómo las representaciones de los problemas limitan lo que se identifica como relevante, dan forma a la comprensión que las personas tienen de sí mismas y sus problemas, e impactan materialmente en sus vidas (pregunta 5).

- una conciencia más aguda de la impugnación que rodea a la representación del "problema" (pregunta 6).

Aplicar las seis preguntas a las propias concepciones e interpretaciones da cuenta de un compromiso que implica incluirse a uno mismo y al propio pensamiento como parte del "material" a analizar. El argumento aquí es que las formas en que se constituyen los "problemas" provocan formas de subjetividad particulares que influyen en cómo nos vemos a nosotros mismos y a los demás. Por tanto, la autoproblematización ("reflexividad") forma parte crucial del análisis.

Desde esta perspectiva, la política pública no es el mejor esfuerzo del gobierno para resolver 'problemas'. Muchas veces, por el contrario, las políticas producen problemas, creando significados particulares que afectan lo que se hace o no se hace, y cómo las personas viven sus vidas. Sin embargo, el enfoque CRP no está centrado en el análisis de la manipulación intencional de problemas o en su marco estratégico. En vez de eso, el enfoque se propone comprender las políticas, mejor que los responsables de su formulación, al analizar los supuestos no examinados y las lógicas conceptuales profundamente arraigadas dentro de las representaciones implícitas del problema. Este enfoque implica prestar atención a las lógicas de conocimiento que están a la base de las políticas públicas - como las premisas psicológicas o biomédicas - y que producen una concepción amplia de gobernar que incluye a expertos y profesionales.

Desde esta perspectiva, el "público", del que somos parte, no se rige a través de políticas, sino a través de problematizaciones: cómo se constituyen los "problemas". Para ser claros, esta afirmación no ignora la multitud de condiciones preocupantes en la vida de las personas (y pueblos); tampoco sugiere que estemos simplemente hablando de interpretaciones, despreciando las condiciones materiales de la vida de las personas. Por el contrario, se propone que las vidas se viven de formas específicas debido al impacto modelador de las comprensiones de los "problemas". Por tanto, el análisis aquí propuesto contradice la suposición relativista de que "una verdad" es tan buena como cualquier otra. 
El enfoque CRP tiene un amplio campo de aplicación. Las leyes específicas o los pronunciamientos de políticas proporcionan los puntos de partida más obvios para el análisis. Sin embargo, los documentos gubernamentales más generales también contienen representaciones implícitas de problemas. Por ejemplo, el compromiso declarado con la promoción de la 'cohesión comunitaria' en un informe gubernamental implica que hay una falta de este estado o condición presuntamente deseable en la comunidad (es decir, la falta de cohesión comunitaria se constituye como un "problema"). Los instrumentos gubernamentales, como los censos o los regímenes de actividad para los desempleados, también pueden analizarse para revelar los supuestos subyacentes sobre qué es problemático y qué necesita cambiar. Además, el enfoque CRP facilita una forma de pensamiento crítico que se extiende mucho más allá del estudio del gobierno y las políticas públicas. Por ejemplo, las seis preguntas resultan útiles para identificar los presupuestos subyacentes y las formas de problematización en las proposiciones teóricas y metodológicas, que de hecho son "soluciones" postuladas.

Inicialmente, el enfoque del análisis de políticas descrito anteriormente se describió como el “¿Cuál es el problema?” (Bacchi, 1999). Quedó claro que se necesitaba una ampliación debido a la tendencia de algunos lectores a interpretar esta pregunta como una determinación de buscar el "problema real" con el fin de desarrollar "soluciones" “apropiadas”. El acrónimo CRP, usado para abreviar “¿Cuál es el problema que se representa?" (por lo que agradezco a Angelique Bletsas), pretende dejar en claro que el objetivo del análisis es comenzar con las 'soluciones' postuladas, por ejemplo en las políticas, para desentrañar y examinar críticamente sus representaciones implícitas de los problemas. Al mismo tiempo, la pregunta 4 abre un espacio para imaginar futuros diferentes, pero siempre con el compromiso de examinar propuestas para sus modos de problematizar.

La encarnación más reciente del enfoque CRP (Bacchi, 2009) incluye dos preguntas (preguntas 3 y 6) que no aparecieron en su formulación inicial. El objetivo de estas preguntas es desarrollar una conciencia más aguda de las formas de poder involucradas en la configuración de las representaciones de los problemas. Un rastreo genealógico del surgimiento de formas particulares de problematización, suscitado por la pregunta 3 , también resalta los espacios de desafío y cambio.

Estas elaboraciones señalan que el enfoque CRP debe concebirse como un modo abierto de compromiso crítico, más que como una fórmula. A la luz de este entendimiento, recientemente: 
- he hecho algunas 'preguntas difíciles' sobre la noción de reflexividad (Bacchi, 2011)

- indagué en el potencial analítico del concepto 'prácticas discursivas' (Bacchi y Bonham, 2011)

- consideré más plenamente las implicaciones políticas de diferentes paradigmas analíticos (Bacchi y Rönnblom, 2011).

En una era en la que la resolución de problemas es el mantra casi hegemónico (piense aquí en la política social basada en la evidencia y en el afán occidental contemporáneo de producir estudiantes que sean 'solucionadores de problemas'), el enfoque CRP sirve como una interrupción muy necesaria a la presunción de que los "problemas" son puntos de partida fijos y no controvertidos, para el desarrollo de las políticas. Nos recuerda que la noción banal y vaga de "el problema”, y su socia, "la solución”, están muy cargados de significado. Para poner a prueba estos significados, el enfoque CRP recomienda el escrutinio de los problemas como una forma de práctica crítica.

\section{Referencias}

Bacchi, C. (1999) Women, Policy and Politics: The construction of policy problems, London: Sage.

(2009) Analysing Policy: What's the problem represented to be? Frenchs Forest: Pearson Education.

(2011) 'Gender mainstreaming and reflexivity: Asking some hard questions', keynote address at the Advancing Gender+ Training in Theory and Practice Conference: An international conference for practitioners, experts and commissioners in Gender+ Training, Complutense University, Madrid, 3 February.

Bacchi, C. and J. Bonham (2011) 'Reclaiming discursive practices as an analytic focus: political implications’, forthcoming.

Bacchi, C. and M. Rönnblom (2011) 'Feminist Discursive Institutionalism - What's Discursive About It? Limitations of conventional political studies paradigms', forthcoming. 


\section{Biografía de la autora}

Carol Lee Bacchi es una cientista política canadiense - australiana, Profesora Emérita de Política en la Facultad de Artes de la Universidad de Adelaida, Australia. Su producción académica se vincula principalmente a la teoría política feminista y políticas públicas. Fue la primera mujer contratada por la universidad en el Departamento de Política de su universidad, y fue distinguida como parte de la Academia de Ciencias Sociales en Australia en 2000. Dentro de sus publicaciones más difundidas destacan: Same difference: Feminism and sexual difference (Sydney, Allen \& Unwin, 1990), The Politics of Affirmative Action: “Women”, Equality and Category Politics (London, Sage, 1996), Women, Policy and Politics: The construction of policy problems (London, Sage, 1999), Fear of Food: A Diary of Mothering (Sydney, Spinifex Press, 2003), Analysing Policy: What's the problem represented to be? (Frenchs Forest, Pearson Education, 2009), Mainstreaming Politics: Gendering practices and feminist theory (with Joan Eveline; Adelaide, University of Adelaide Press, 2010) and Poststructural Policy Analysis: A Guide to Practice (with Susan Goodwin; New York, Palgrave Macmillan, 2016). Perfil académico: https://researchers.adelaide.edu.au/profile/carol.bacchi Sitio web: https://carolbacchi.com/ 